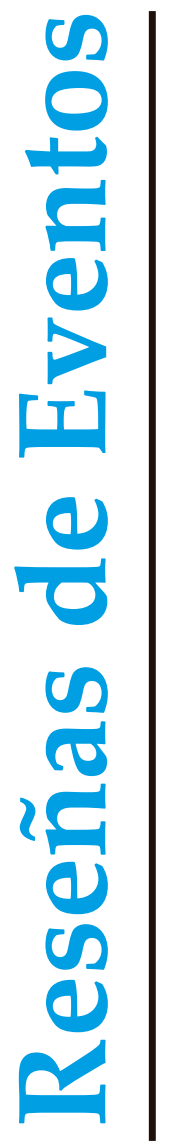

\title{
Eventos
}

\section{Reseñas Reseñas}

Reseñas Reseñas de Eventos

\section{Reseñas de Eventos}

\section{Reseñas de Eventos}

Reseñas de Eventos

Reseñas de Eventos
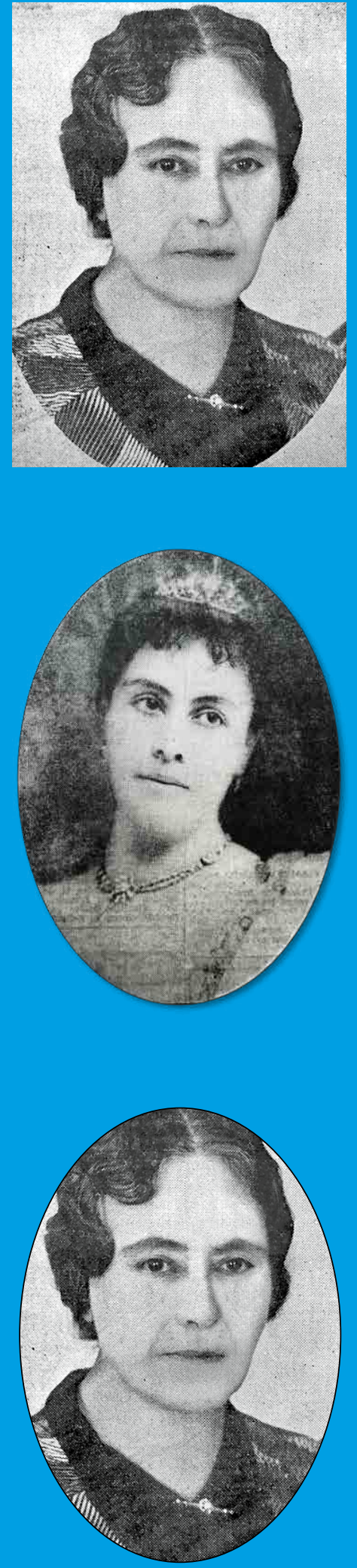



\title{
XVIII CONGRESO DE LA FEDERACIÓN INTERNACIONAL DE ESTUDIOS SOBRE AMÉRICA LATINA Y EL CARIBE (FIEALC),
}

\author{
Belgrado - Serbia, 25 - 28 de julio de 2017 \\ SIMPOSIO
}

"El futuro de la Universidad Latinoamericana y del Caribe a debate. Desafíos y nuevas perspectivas en la identidad intercultural"
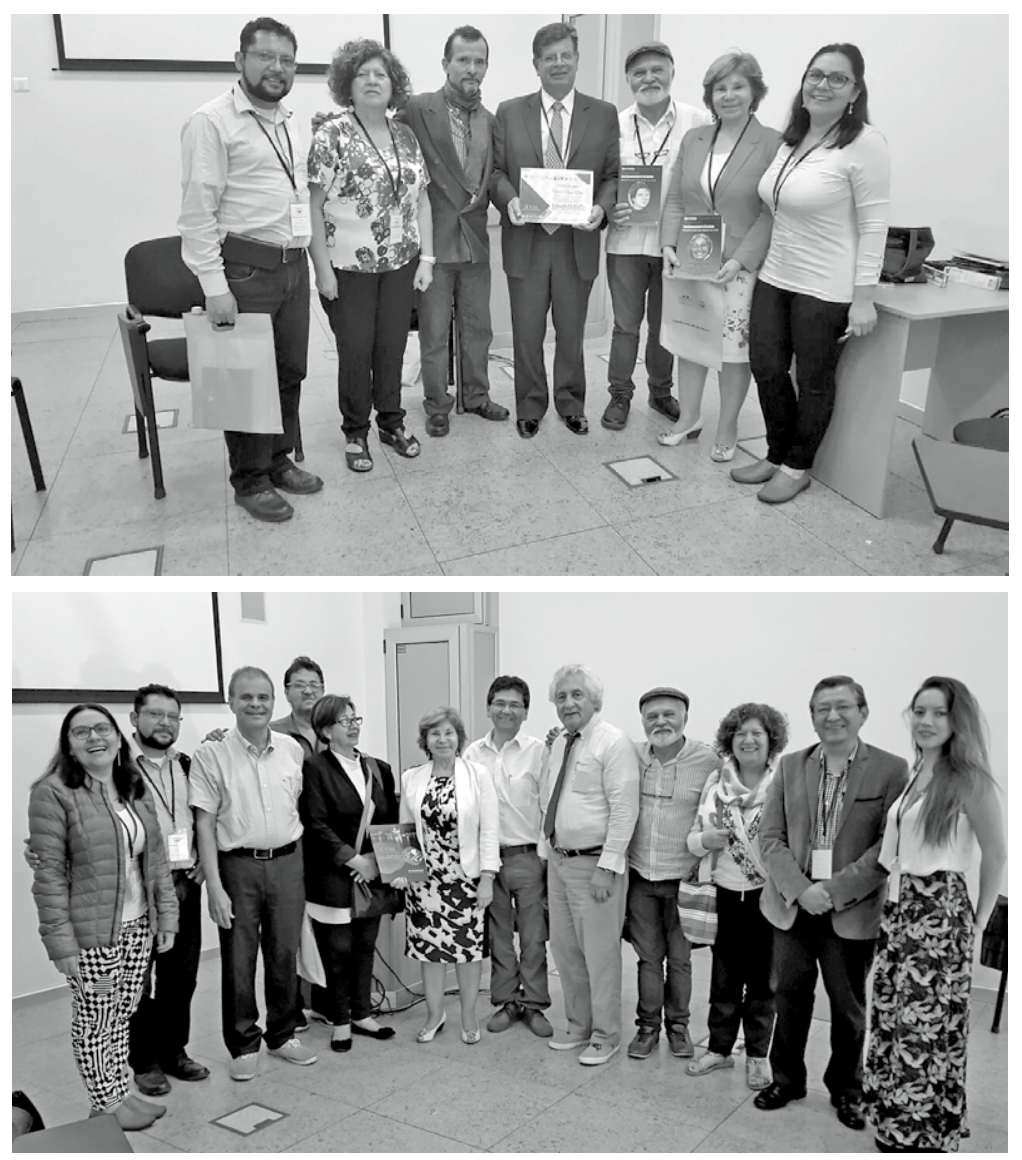

El XVIII Congreso de la Federación internacional de estudios sobre América Latina y el Caribe (FIEALC), se llevó a cabo en la Universidad Megatrend "John Naisbitt", del 25 al 28 de julio de 2017, en Belgrado (SERBIA). En esta ocasión se realizó en homenaje a la maestra María Elena Rodríguez Orzán. Al evento asistieron 
188 investigadores de 44 países y fue coordinado por el Dr. Slobodan S. Pajović. Dentro de este evento se desarrolló el Simposio "El futuro de la Universidad Latinoamericana y del Caribe a debate. Desafíos y nuevas perspectivas en la identidad intercultural", en el cual presentaron ponencia 18 investigadores de seis países. Las ponencias se clasificaron en cuatro grandes temáticas: La primera, la prospectiva; la segunda, la formación en las universidades; la tercera, sobre Neoliberalismo, administración, tecnologías e inclusión. La cuarta, Nuevas miradas hacia los Estudios Superiores. El tema se centró en un diálogo continuo de generaciones que confluyen con la experiencia, la creatividad y especialmente una identidad con ética y responsabilidad social. El simposio estuvo bajo la dirección del Grupo de Investigación "Historia y Prospectiva de la Universidad Latinoamericana". HISULA, dentro de la conmemoración de los 25 años de fundación del grupo, que en este tiempo ha coordinado eventos en 27 países.

Por este motivo, este evento académico se centró en la visión sobre la prospectiva de la universidad Latinoamericana en el siglo XXI, en concordancia con la línea de investigación que viene trabajando el grupo desde el año 2006. Es así que se han realizado investigaciones sobre las políticas supranacionales hacia las universidades latinoamericanas. Se ha revisado la visión actual de las grandes universidades del mundo, pero fundamentalmente nos centramos en las dinámicas de la nueva generación que está ingresando a nuestras instituciones universitarias y que las estarán dirigiendo en aproximadamente diez años. Pero a su vez, reflexionamos sobre cuál es nuestro legado, y qué decisiones hemos tomado frente a esos retos que, en el año 1963, nos puso en la palestra Darcy Ribeiro con respecto a la identidad Latinoamericana.

Por supuesto, entendemos que las relaciones sociales se desenvuelven en un mundo globalizado, pero a su vez, reconocemos unas raíces que nos hacen diferentes y ese aspecto fundamental será el motor que hará realidad las transformaciones que nos propongamos para nuestro continente americano. Esperamos que este tema de la prospectiva universitaria se revierta en un diálogo continuo de generaciones en el que confluya la experiencia, la creatividad y especialmente una identidad con ética y responsabilidad social. La dirección de este Simposio estuvo a cargo de los investigadores Dra. Diana Elvira Soto Arango, de la Facultad de Educación de la Universidad Pedagógica y Tecnológica de Colombia, Dr. Armando Martínez de la Universidad de Guadalajara México, Dr. José Rubens Lima Jardilino de la Universidad de Ouro Preto, Brasil, y Dr. José Enrique Cortez Sic de la USAC de Guatemala.

Por otra parte, durante el Congreso se efectuó la presentación de publicaciones donde se subrayó la categoría científica de la Revista Historia de la Educación Latinoamericana. -RHELA- y las publicaciones producto de redes de investigación como la que se lidera sobre "Educadores latinoamericanos". Asimismo, hizo presencia la junta directiva de la Sociedad Historia de la Educación LatinoamericanaSHELA-, que desarrolló una reunión donde apoyó una vez más las actividades del Doctorado Ciencias de la Educación de CADE-UPTC- RUDECOLOMBIA y en esta ocasión se amplió la colaboración a los proyectos de investigación, publicaciones y movilidad de la Facultad de Educación de la Universidad Pedagógica y Tecnológica deColombia. Por otra parte, se establecieron relaciones académicas y de investigación 
con otras instituciones internacionales vinculadas a la FIEALC que se revertirán en proyectos y publicaciones conjuntas.

Finalmente indicamos que, en la Asamblea de la FIEALC, el Presidente del Congreso resaltó este Simposio como el mejor organizado del evento y además destacó la presencia del señor rector Alfonso López de la Universidad Pedagógica y Tecnológica de Colombia, en calidad de conferenciante. Por otra parte, la Sociedad Historia de la Educación Latinoamericana -SHELA-, realizó una reunión de la junta directiva donde se planificaron las actividades de investigación y de publicaciones para el 2018 y se dio un reconocimiento público a la labor desarrollada por el joven investigador Diego Naranjo del grupo HISULA y al equipo coordinador del simposio.

Elaboró: Dra. Diana Elvira Soto Arango

Decana Facultad de Educación Uptc

Grupo de investigación HISULA

\section{DIPLOMADO EN PEDAGOGÍAS E INTERCULTURALIDAD Resguardo Unido U'wa, agosto de 2017}
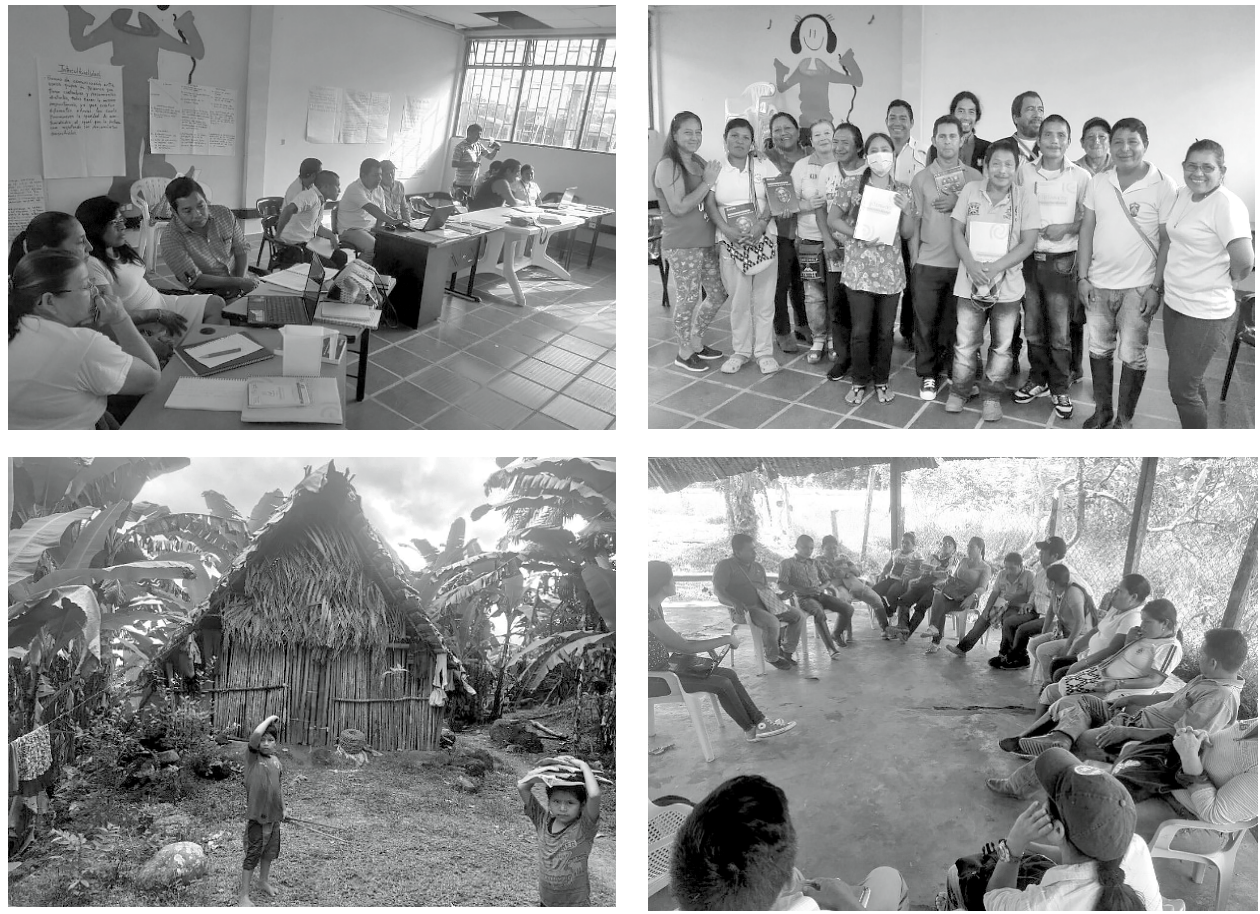

Fuente: Diego Naranjo, agosto 2017. Resguardo Unido U’wa. 
Diplomado en Pedagogías e Interculturalidad es un proyecto educativo de extensión, formación y cualificación de educadores/as rurales apoyado por los proyectos de investigación, que desarrolla el grupo de investigación Historia y Prospectiva de la Universidad Latinoamericana-HISULA-, avalado por el comité de currículo de la Escuela de Licenciatura en Ciencias Sociales en sesión del 15 de mayo de 2017; Consejo de Facultad de Ciencias de la Educación (17 de mayo de 2017), presentada por el CIEFED y viabilizada por el Comité Territorial de Formación Docente de la Secretaría de Educación de Boyacá, en sesión del 16 de junio de 2017, como créditos de ascenso en el Escalafón Nacional Docente, según el Decreto 1278 de 2002.

La primera versión del diplomado se desarrolló bajo la modalidad presencial, con una intensidad horaria de 336 horas, equivalentes a 7 créditos académicos en Colombia, en agosto de 2017. Se impartió a etnoeducadores de la comunidad U'wa, Resguardo Unido U'wa en el departamento de Cubará, Boyacá. Institución Educativa Técnica Pablo VI y Escuela Indígena El Chuscal. Esta comunidad presenta 16 núcleos educativos para los 3700 habitantes.

El diplomado se desarrolló en tres módulos, en el módulo 1 se abordó el concepto de interculturalidad ${ }^{1}$ en la educación, aplicando como dispositivos didácticos, los círculos de reflexión, desde la corriente historiográfica de la línea de investigación y de formación de docentes e interculturalidad, del Grupo de HISULA, con el enfoque teórico-metodológico a través del desempeño y liderazgo educativo del educador, que se interrelaciona con los contextos sociopolítico-culturales. De esta manera, los participantes a partir del diálogo, la lectura grupal y el análisis de contenido audiovisual se acercaron al proceso histórico del principio de la interculturalidad en la educación que ha sido incorporado y adaptado desde las propuestas educativas inclusivas, alternativas, constructoras de nuevas epistemes pedagógicas y didácticas en contextos de la escuela situada.

Estas consideraciones permitieron a los maestros participantes reconocerse como actores sociales, políticos e históricos en unos espacios de poder local y nacional ${ }^{2}$, y agentes culturales con la capacidad de resignificar su labor y configurarse como intelectuales comprometidos con la pedagogía social y la educación intercultural.

En el módulo 2, a partir del componente pedagógico de la propuesta, y el desarrollo de actividades de interpretación audiovisual, lectura compresiva y la técnica de grupo focal, los participantes lograron avanzar en el reconocimiento de tendencias pedagógicas, como la pedagogía de la potenciación, afines al contexto

1 Sustentado en Diana Elvira Soto Arango, Diego Eduardo Naranjo Patiño, Justo Cuño Bonito. Historia de vida de educadores. Aproximación historiográfica y metodológica, Tunja, Universidad Pedagógica y Tecnológica de Colombia, HISULA, 2017). Inédito. Soportado en los proyectos de investigación: La Formación de las maestras rurales en Colombia y Brasil: Perspectivas de estudios comparados en educación SGI 1730 Uptc, Maestras africanas y afrodescendientes en Colombia, Guatemala, Brasil, Venezuela, Jamaica y Guinea Ecuatorial siglos XX al XXI SGI 1732 Uptc: Formación de educadores en las escuelas normales superiores convenio UPTC. 2010-2017» SGI: 2192, el de "Historias de vida de maestras rurales, indígenas y afrodescendientes. Un estudio comparado en los municipios de Soracá, Puerto Boyacá y Güicán de la Sierra"

2 Soto Arango, Diana Elvira, La Escuela Rural En Colombia. Historias de Vida de Maestras. Mediados del Siglo XX. Tomo VI. Colección Educadores.(Tunja, Colombia, 2014). 
particular de las comunidades rurales e indígenas en donde la apropiación de la investigación, se asume como una estrategia pedagógica para la transformación de la realidad educativa y la defensa de los derechos culturales.

En el módulo 3 se lograron definir los principios de la investigación educativa, como mecanismo a través del cual las comunidades se empoderan en su quehacer, los maestros resignifican su labor y apropian la organización y dinamización educativa como ejes transversales del avance de la comunidad en la consecución de sus objetivos como nación. Como herramientas didácticas se propuso la matriz DOFA, el árbol de problemas y la metodología de formulación de proyectos de investigación. Como resultado del proceso de formación se adelantaron cuatro proyectos: uno audiovisual que da cuenta de las historias de vida, el proceso de escolarización del territorio U'wa; un programa de radio, como mecanismo para empoderar y visibilizar la labor que adelantan los etnoeducadores en sus contextos; la formulación de un programa para la formación de etnoeducadores, que garantice el relevo generacional, y por último un proyecto encaminado al diseño de material didáctico y pedagógico pertinente y coherente con las características del territorio.

En definitiva, se partió de la concepción del educador que entendemos como un actor social en unos espacios de poder local y nacional ${ }^{3}$. En tal sentido, el punto central del enfoque identifica al educador como una persona que transforma realidades y con responsabilidad social en contextos socio-culturaleseducativos, coherentes con un modelo pedagógico, donde se indaga el "acto del conocimiento que será transformado por el educador y el educando" (Soto Arango, 2017).

El trabajo desarrollado representa para esta comunidad educativa un acercamiento a los procesos académicos y de investigación adelantados por el grupo de investigación Historia y Prospectiva de la Universidad Latinoamericana -HISULA-, de la Universidad Pedagógica y Tecnológica de Colombia. Así mismo, consideramos que se garantiza y permite el reconocimiento de la política de extensión universitaria dentro del principio de responsabilidad social de la institución.

Diego Eduardo Naranjo Patiño Universidad Pedagógica y Tecnológica de Colombia Grupo de investigación HISULA

3 Soto-Arango Diana Elvira. Cuño Justo. López O. H. La maestra rural en Iberoamérica. Historias de vida de maestras. Tomo VIII. Sevilla, (Tunja, Universidad Pablo de Olavide. España, Universidad Pedagógica y Tecnológica de Colombia. Universidad San Carlos de Guatemala, 2015). 


\section{EGRESADO DEL DOCTORADO EN CIENCIAS DE LA EDUCACIÓN RUDECOLOMBIA- UPTC OBTIENE PREMIO ORDEN CÍVICA CIUDAD DE CHIQUINQUIRÁ 2017 Chiquinquirá-Colombia, 1 de septiembre de 2017}

El graduado del Doctorado en Ciencias de la Educación Rudecolombia-UPTC doctor Jorge Enrique Duarte Acero, obtuvo el pasado viernes $1^{\circ}$ de septiembre del año en curso, el premio "Orden Cívica Ciudad de Chiquinquirá en el Grado de Oficial". La Orden Cívica fue creada mediante Decreto Número 035 de 1991 (marzo 8) "con el propósito de reconocer a las personalidades, instituciones y gremios que han trabajado en favor de la capital religiosa de Colombia".

La "Orden Cívica Ciudad de Chiquinquirá en el Grado de Oficial" fue impuesta por el Alcalde Municipal, doctor César Augusto Carrillo Ortegón al doctor Duarte Acero en la Conmemoración de los 207 años de la fundación de la Ciudad de Chiquinquirá como Villa Republicana. La orden fue Otorgada por la Alcaldía Municipal de Chiquinquirá, previo consentimiento del Consejo de Gobierno y el Concejo Municipal, por el Decreto número 101 de 2017 (septiembre 01) y que en el Considerando expresa: “3. Que el doctor Jorge Enrique Duarte Acero, docente chiquinquireño, se ha destacado en la investigación en las áreas de formación de educadores del siglo XIX y XX, con alta experiencia en el tema administrativo y educativo".

El docente upetecista Jorge Enrique Duarte Acero está vinculado actualmente con la Facultad de Ciencias de la Educación, Escuela de Psicopedagogía y ejerce la Coordinación Académica de los Programas de Posgrado de Especialización en Gerencia Educacional y de la Maestría en Gestión Educativa.

Elaboró: Sandra Liliana Bernal Villate Grupo de investigacion HISULA 


\section{PRIMER ENCUENTRO INTERNACIONAL DE INVESTIGACIÓN UNIVERSITARIA}

Tunja - Colombia, 7 y 8 de septiembre de 2017

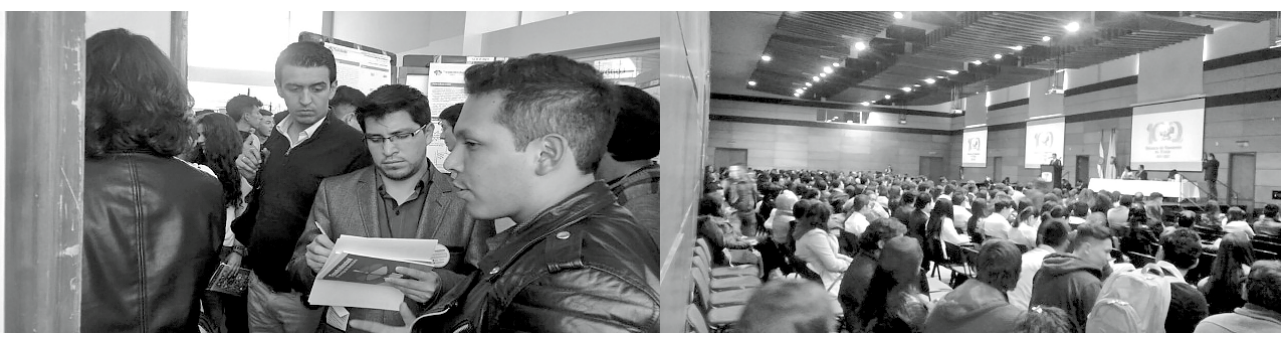

La Universidad Santo Tomás y la Universidad Pedagógica y Tecnológica de Colombia realizaron el Primer Encuentro Internacional de Investigación Universitaria (EnIIU) que se llevó acabo en el Centro de Convenciones de la Cámara de Comercio en la ciudad de Tunja (Boyacá - Colombia) los días 7 y 8 de septiembre de 2017.

Este evento contó con la intervención de investigadores de distintas Universidades y la participación de tres conferencias magistrales, junto con 253 ponencias en las categorías de: arquitectura, ingenierías, derecho, humanidades, ciencias administrativas, salud, ciencias básicas, ciencias de la educación, ciencias agropecuarias, y 109 posters en esas mismas categorías. El grupo Historia y Prospectiva de la Universidad Latinoamericana- HISULA- (UPTC) participó con los semilleros de investigación y sus propuestas de impacto social, en el contexto educativo desde diferentes perspectivas que analizaron las proyecciones pedagógicas, culturales y sociales. La actividad contó con la participación de los jóvenes investigadores HISULA; Diego Eduardo Naranjo como jurado evaluador y Luis Eduardo Molina como organizador logístico.

La coordinación del evento estuvo a cargo de la organización de las instituciones anfitrionas; Universidad Santo Tomás (USTA), Universidad Pedagógica y Tecnológica de Colombia (UPTC) y tuvo como objetivo impulsar, en la comunidad estudiantil, la investigación de índole formativa y participativa en los niveles de pregrado y maestría.

Elaboró: Paola Andrea Ochoa Pérez Sandra Milena Ochoa Pérez Universidad Pedagógica y Tecnológica de Colombia Semilleros de investigación - Grupo HISULA 